\title{
Description de la larve et considérations sur l'habitat, la biologie et la répartition de Thremma tellae (Trichoptera : Uenoidae : Thremmatinae).
}

\author{
M.A Gonzálezl \\ J.C. Iglesias 1 \\ F. Cobol
}

Mots clés : Trichoptera, Thremmatinae, Thremma, larves, morphologie, biologie, Péninsule Ibérique.

La larve de Thremma tellae est décrite pour la première fois, par comparaison avec celle de Thremma gallicum, dont elle se distingue nettement par quelques particularités morphologiques. Son fourreau et son comportement constructeur constituent les caractères les plus originaux. Les auteurs donnent également quelques remarques biogéographiques et écologiques.

A description of the larva of Thremma tellae (Trichoptera : Uenoidae : Thremmatinae) with notes on its habitat, biology and distribution.

Keywords: Trichoptera, Thremmatinae, Thremma, larvae, morphology, biology, Iberian Peninsula.

The larva of Thremma tellae is described for the first time and compared with that of Thremma gallicum, from which it differs in a distinctive set of characters. The most striking feature of $T$. tellae is the building behaviour of the larva. Some zoogeographical and ecological notes are included.

\section{Introduction}

Thremma est un petit genre à répartition circumméditerranéenne, dont la position systématique, discutée depuis longtemps, vient d'être révisée récemment par Vineyard \& Wiggins (1988). A ce jour il compte cinq espèces, peuplant des aires géographiques relativement limitées : $T$. sardoum Costa 1884 , qui en dehors de la Corse-Sardaigne se trouve en Algérie ; T. anomalum McLachlan 1876, espèce du Sud-Est d'Europe ; T. martynovi Malicky 1976, espèce du Caucase et d'Asie Mineure ; T. gallicum McLachlan 1880, espèce d'Europe occidentale, rencontrée dans la Péninsule Ibérique, où son aire de répartition recouvre partiellement celle de $T$. tellae González 1978, un endémique ibérique (González, 1978).

Seules trois espèces sont bien connues à l'état larvaire : $T$. gallicum, $T$. sardoum et $T$. anomalum. Les descriptions de Jacquemart \& Coineau (1966)

1. Departamento de Biología Animal, Facultad de Biología, Universitad de Santiago, 15706 Santiago de Compostela, España. première espèce et celles de Giudicelli (1971) aux deux premières ; enfin, Botosaneanu (1956) fournit une description détaillée de la larve de $T$. anomalium. Les stades aquatiques de $T$. martynovi et $T$. tellae restent inconnus à ce jour.

Lors de chasses trichoptérologiques dans le NordOuest de la Péninsule Ibérique nous avons récolté d'étranges constructions ayant l'aspect de petites mûres et renfermant des larves et des nymphes prêtes à émerger. Les adultes obtenus appartenaient à l'espèce $T$. tellae. Nous avons récolté aussi, souvent dans les mêmes localités, de nombreuses larves et nymphes de $T$. gallicum. Nous donnons ici une courte description de la larve de $T$. tellae, limitée aux caractères discriminants de ceux de $T$. gallicum.

\section{Description}

\subsection{LA LARVE}

Elle présente l'habitus caractéristique des Thremma. Au dernier stade la taille maximale de la larve est de $6.0 \mathrm{~mm}$. Capsule céphalique légèrement plus longue que large en vue dorsale $(\mathrm{fig} .1 \mathrm{~A})$; le rapport moyen longueur-largeur (pièces buccales 
non comprises) est de 1.150. Chez $T$. gallicum (fig. I D) la capsule céphalique est à peu près aussi longue que large (rapport moyen longueur-largeur : 1.025).

La coloration de la capsule céphalique présente une différence marquée au niveau de la région occipitale. Chez $T$. tellae il y a une grande tache blanche de part et d'autre de la suture coronale, alors que chez $T$. gallicum cette zone présente toujours deux grosses taches pigmentées et le plus souvent accompagnées de quelques taches plus petites. De plus, on trouve le long de sa suture ventrale une bande pâle (fig. $1: \mathrm{H}, \mathrm{J}$ ), plus large chez $T$. gallicum que chez $T$. tellae. En dehors de ces caractères de coloration on peut remarquer aussi, chez $T$. tellae, la présence sur les genae, des deux côtés du submentum, d'une série de petites soies (une dizaine environ); ces soies ne semblent pas se retrouver chez T. gallicum (fig. 1 : I, K).

Les tergites thoraciques de $T$. tellae ne montrent aucune particularité remarquable par rapport aux autres espèces. Cependant il faut signaler que, chez T. tellae, la pilosité du pronotum est, dans l'ensemble, beaucoup plus dense que chez $T$. gallicum (fig. $1:$ B, F). De plus, l'aspect des sclérites du mesonotum (fig. $1: \mathrm{C}, \mathrm{G}$ ), bien qu'assez variable, permet de distinguer les deux espèces. Cette différence est particulièrement évidente au niveau des sclérites médians antérieurs ; chez $T$. tellae les bords internes de ces sclérites sont nettement divergents alors que chez $T$. gallicum ils sont étroitement rapprochés de la ligne médiane.

L'examen des pattes antérieures (même chez les larves les plus jeunes) fournit le caractère le plus net pour distinguer facilement la larve de $T$, tellae des autres espèces. Ce caractère est visible au niveau des fémurs ( fig. 2 D) qui, en vue latérale, sont larges et trapus avec une forte convexité du bord supérieur. Compte tenu du manque d'iconographie ayant trait à ce caractère pour $T$. sardoum et $T$. anomalum, nous avons figuré les pattes antérieures de ces deux espèces (fig. $2: \mathrm{B}, \mathrm{C}$ ).

Le premier segment abdominal possède, comme chez les autres espèces du genre, un grand mamelon ventral pourvu de deux sclérites de contour irrégulier et légèrement variable (fig. 1Q) ; l'aspect de ces sclérites permet de distinguer assez aisément les larves de $T$. tellae de celles de $T$. gallicum -comparer notre fig. $1 \mathrm{Q}$ avec la fig. 8 A de Giudicelli (1971).
Schéma des branchies :

\begin{tabular}{|c|c|c|c|c|c|}
\hline \multirow{2}{*}{ Dorsal : } & 0 & 0 & 0 & $0-1$ & 0 \\
\hline & $2-3$ & $2-3$ & $2-3$ & 3 & $2-3$ \\
\hline \multirow{2}{*}{ Ventral : } & 0 & $0-1$ & 1 & $0-1$ & 0 \\
\hline & 3 & $2-3$ & $2-3$ & $2-3$ & $2-3$ \\
\hline
\end{tabular}

\subsection{LE FOURREAU}

La structure du fourreau de $T$. tellae (fig. 3) est extrêmement intéressante ; les larves construisent d'abord un fourreau exclusivement minéral - pas du type ancyliforme, comme chez $T$. gallicum et $T$. sardoum - mais conique et recourbé (comme chez T. anomalum), à l'aide de grains de sable de taille variable ; 1'ouverture antérieure est grande et oblique, et l'orifice postérieur, plus étroit, est partiellement obturé par une membrane operculaire, de soie percée d'un petit orifice circulaire. Cet étui minéral, qui peut atteindre $7,5 \mathrm{~mm}$ de longueur, est recouvert dorsalement (même aux stades jeunes), par de petites boulettes confectionnées par la larve. Ces boulettes, de taille variable, sont constituées par l'assemblage de débris organiques, de microphytes, de fins éléments minéraux... reliés entre eux par de la soie. En ce qui concerne la densité des boulettes formant ce revêtement original, densité qui dépend probablement de la proportion dans le milieu des éléments nécessaires à sa réalisation, on trouve une certaine variabilité ; ajoutons que ce revêtement est parfois composé à la fois de boulettes et de petits grains de sable.

Nos données relatives au comportement constructeur sont encore fragmentaires. A l'aide d'une loupe binoculaire nous avons observé épisodiquement, au laboratoire, certains détails concernant la confection des boulettes. La larve, à l'aide des pièces buccales et des pattes antérieures, amasse des éléments du substrat qui sont agglomérés entre eux par de la soie. Ces éléments, manipulés avec une grande habileté, forment alors une boulette que la larve fixe ensuite par des fils de soie à la construction minérale (la durée des diverses séquences dont nous venons de parler est de l'ordre de quelques minutes). Il est intéressant de noter que les boulettes semblent être assez lâchement fixées, car elles se détachent facilement ne résistant pas aux légères manipulations du fourreau. On peut alors supposer que le renouvellement des boulettes doit être continu tout au long de la vie 

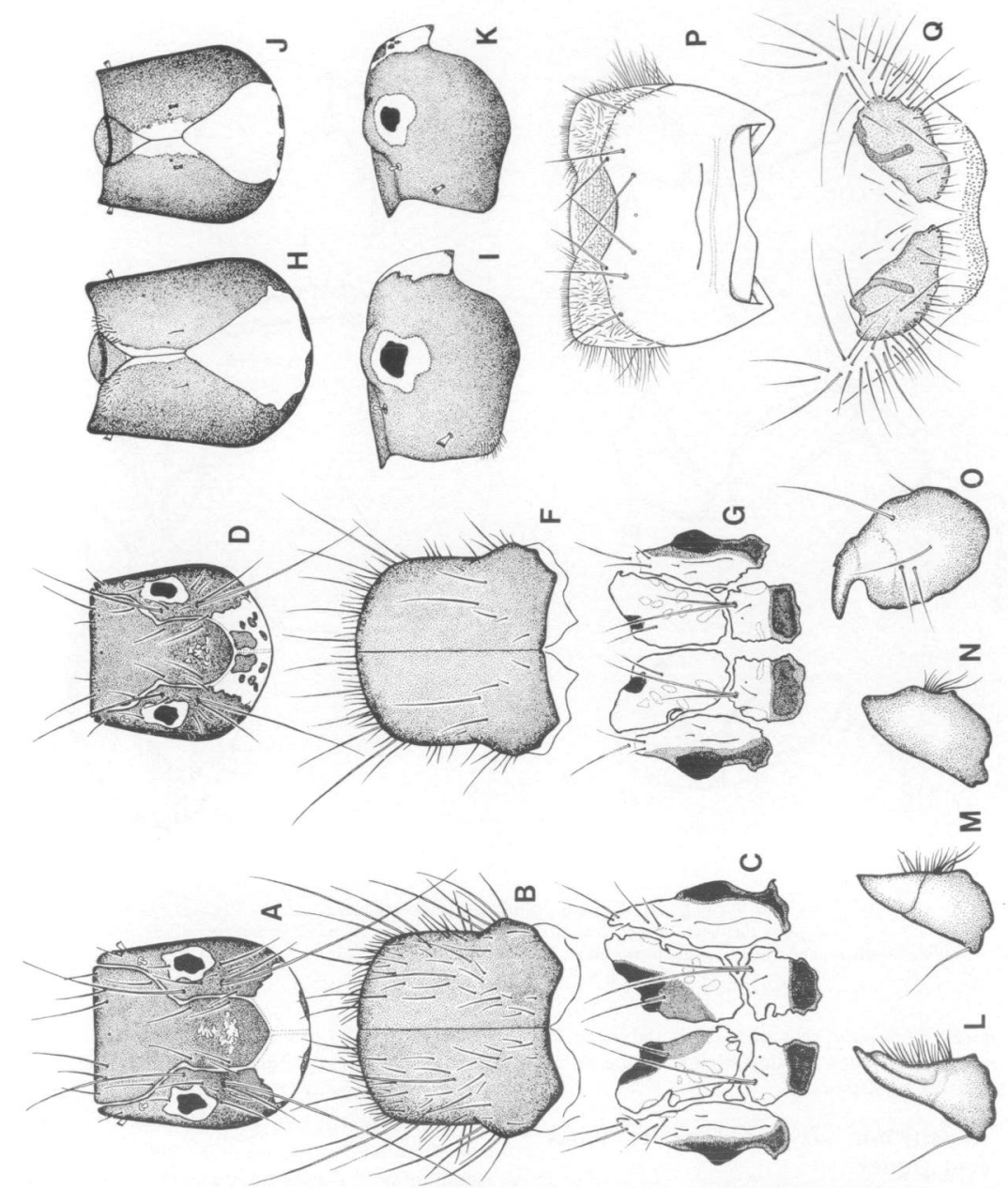

Fig. 1. Thremma tellae Gonz. et Thremma gallicum McL., larves.

Thremma tellae : A. capsule céphalique, vue dorsale. B. pronotum, vue dorsale. C. sclérites du mesonotum, vue dorsale. H. capsule céphalique, vue ventrale. 1 . capsule céphalique, vue latérale. L. mandibule droite, côté ventral. M. mandibule gauche, côté dorsal. N. mandibule gauche au dernier stade d'usure, côté dorsal. O. Crochet anal droit, face latérale. P. labrum, vue dorsale. Q. sclérites du mamelon ventral du premier segment abdominal.

Thremma gallicum : D. capsule céphalique, vue dorsale. F. pronotum, vue dorsale. G. sclérites du mesonotum, vue dorsale. I capsule céphalique, vue ventrale, $K$. capsule céphalique, vue latérale. 


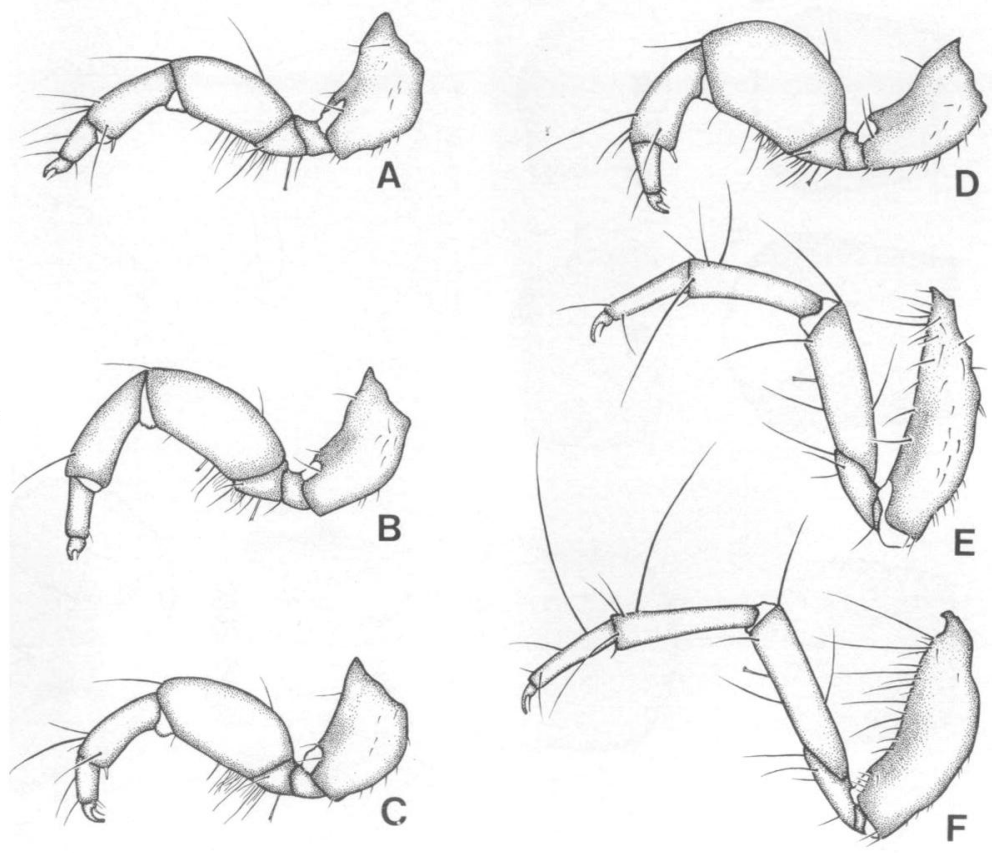

Fig. 2. Thremma spp., pattes des larves, vue latérale.

A. patte antérieure, $T$. gallicum. B. idem, T. sardoum. C. idem, T. anomalum.

D-F. T. tellae ; D. patte antérięure ; E. patte intermédiaire ; F. patte postérieure.

larvaire ; c'est pour cela que dès le début de la nymphose, les fourreaux nymphaux ont perdu la plupart des boulettes.

\section{Répartition géographique et notes écologiques}

T. tellae est un endémique de la Péninsule Ibérique dont la répartition est surtout limitée au quadrant nord-occidental. Dans cette région l'espèce a été trouvée dans de nombreuses localités de la Galice et des provinces limitrophes (León, Zamora), au nord et au centre du Portugal et dans plusieurs provinces du centre de l'Espagne (Madrid, Segovia, Avila et Cáceres). L'aire de répartition de $T$. gallicum dans la Péninsule est plus étendue ; en plus du quadrant nord-occidental, où les deux espèces cohabitent fréquemment, cette espèce a été signalée dans d'autres provinces (Oviedo, Santander, Palencia, Soria, Zaragoza, Huesca, etc...) de toute la moitié nord de la Péninsule. 


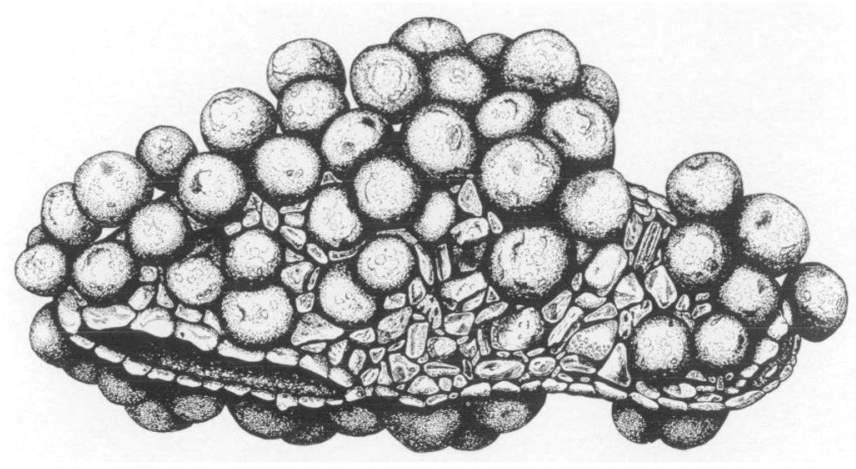

Fig. 3. Thremma tellae, fourreau larvaire au dernier stade, vue latérale.

Nos connaissances sur la biologie et les exigences écologiques de $T$. tellue sont encore fragmentaires. En ce qui concerne sa répartition longitudinale dans les cours d'eaux et sa répartition altitudinale il ne paraît pas y avoir de différences fondamentales entre les deux espèces ibériques. T. tellae présente une vaste répartition altitudinale $(50$ a $1700 \mathrm{~m})$ et une large variété d'habitats ; elle est particulièrement bien représentée dans les sources et ruisseaux de la zone prémontane et montane mais on la trouve aussi dans le cours inférieur de certaines petites rivières (González, 1988). Comme les autres espèces du genre, ses larves pétricoles se déplacent sur le substrat dont elles râclent la couverture biologique : les contenus stomacaux ont révélé des débris végétaux fins, beaucoup de Diatomées et d'autres microphytes. La distribution des larves par rapport au courant est assez variable ; néanmoins, l'espèce semble moins rhéophile que $T$. gallicum. Son habitat le plus fréquent est représenté par les zones calmes ou à courant modéré où les larves pullulent sur la face supérieure des pierres. Elles y sont le plus souvent associées à plusieurs espèces de Polycentropus et de Silo.

\section{Remerciements}

Nous tenons à remercier le $\mathrm{D}^{\mathrm{r}} \mathbf{L}$. Botosaneanu (Amsterdam), dont les critiques ont permis une amélioration du manuscrit original.
Plusieurs collègues nous ont envoyé du matériel de comparaison de leur propre collection, J. Giudicelli (Marseille), K. Kumanski (Sofia) et W. Mey (Berlin). L. Terra (Vila do Conde) et D. García de Jalón (Madrid) nous ont également communiqué une liste de localités de captures.

Que tous trouvent ici l'expression de notre sincère reconnaissance.

Enfin, les auteurs remercient la CICYT (PB 86-0406) qui a subventionné ce travail.

\section{Travaux cités}

Botosaneanu (L.). 1956. - Recherches sur les Trichoptères de Bulgarie recueillis par MM. le Prof. A. Valkanov et B. Rusev. Beit. Z. Entom., 6 (3-4) : 354-402.

Giudicelli (J.). 1971. - Monographie du genre Thremma (Trichoptera, Thremmatidae). Annls Limnol, 7 (1) : 125-139.

González (M.A.). 1978. - Descripción de Thremma tellae nov. sp. (Trich. Thremmatidae) de la Peninsula Ibérica. Bol. Asoc. esp. Entom, 2 : 201-203.

González (M.A.). 1988. - Inventario dos Tricópteros de Galicia (Insecta : Trichoptera). Cad. Area Cienc, Biol. (Inventarios) $2: 1-45$.

Jacquemart (S.) \& Coineau (Y.). 1966. - A propos de Thremma gallicum MacLachlan (Trichoptera). Bull. Inst. r. Sci. nat. Belg, 42 (17) : 1-14.

Vineyard (R.N.) \& Wiggins (G.B.). 1988. - Further revision of the caddisfly family Uenoidae (Trichoptera) : evidence for inclusion of Neophylacinae and Thremmatidae, Syst. Entom., $13: 361-372$. 